\title{
Annual Mean and Correlation of Global Vertical Total Electron Content from Various Global Data Centers
}

\author{
Shambel Gizachew ${ }^{1}$, Belay Sitotaw ${ }^{1}$, Gizaw Mengistu ${ }^{2}$ \\ ${ }^{1}$ Physics Department, Dire Dawa University, Dire Dawa, Ethiopia \\ ${ }^{2}$ Physics Department, Addis Ababa University, Addis Ababa, Ethiopia
}

Email address:

bingiza2015@gmail.com (S. Gizachew)

\section{To cite this article:}

Shambel Gizachew, Belay Sitotaw, Gizaw Mengistu. Annual Mean and Correlation of Global Vertical Total Electron Content from Various Global Data Centers. American Journal of Astronomy and Astrophysics. Vol. 8, No. 1, 2020, pp. 1-7. doi: 10.11648/j.ajaa.20200801.11

Received: November 21, 2019; Accepted: December 31, 2019; Published: January 21, 2020

\begin{abstract}
The main purpose of this work is to evaluate the annual mean correlations of vertical total electron content provided by GPS, Center for Orbit Determination in Europe (CODE), Jet Propulsion Laboratory (JPL), Polytechnical University of Catalonia (UPC) and European Space Agency (ESA) data centers. The comparisons are aimed at comparability of the different vertical total electron content (VTEC) data sets in terms of times variabilities globally. The Annual mean comparison of these global vertical total electron content are as expected results and also the agreements between the different VTECs from the given data centers also assessed from correlation maps is very good as reflected in correlation exceeding 0.98 among the different data sets with the exception of ESA VTEC. Moreover, the result shows that a distinct feature which exhibit by all data sets is presence of peak VTEC along equatorial latitude belt which fades out with increase in latitude.
\end{abstract}

Keywords: Total Electron Content, GPS, Electron Density

\section{Introduction}

The ionosphere is about $60-1000 \mathrm{~km}$ above the earth's surface, which is actually plasma of ionized gas of the upper atmosphere by solar radiation and high-energy particles from the Sun. The ionized electrons concentrations change with height above earth's surface, location, time of the day, season, and amount of solar activity [1]. Usually the ionosphere consists of four layers, the D, E, $F_{1}$ and $F_{2}$ layers, and the peak ionospheric electron density locates at the $F_{2}$ layer. According to the estimated vertical electron density distribution deriving from the Chapman profile, 90 electrons of the whole ionosphere are focusing from $100 \mathrm{~km}$ to $800 \mathrm{~km}$ [2].

Earth's ionosphere is an important part of the near space environment; its condition is determined by the solar radiation fluxes within the different wavelength ranges [3]. Nowadays, ionosphere monitoring is realized by different ground-based and satellite radio sounding instruments [4]. It allows determining of local ionosphere parameters, but they have significant global distinctions. Since, the ionosphere is as a main source of range and range rate errors for users of satellite based radars and navigation system like global navigation positioning system (GPS). In terms of ambient of electron density variations and characteristics of density irregularities, the Earth's ionosphere may be divided in to three latitudes zones: the first zone is the equatorial region covering between $\pm 30^{\circ}$ dip around the magnetic equator, the high latitudes is the region covering latitude above $60^{\circ}$ the dip magnetic pole and the third region is the mid-latitudes region lying in between [5].

When the solar radiation is incident on a gas atom or molecule, the part of this radiation is absorbed by the atom; a free electron and a positively charged ion are produced. At the highest altitude the solar radiation is very strong but there are only few atoms are available to interact, so the ionization is small. As the latitude decreases, the more gas atoms or molecules are present to interact so the ionization is greater. At the same time an opposite process called recombination starts to happen in which a free electron is captured by a nearby positive ion. As the gas density is high at lower altitudes, so the recombination increases at lower altitudes $[2]$.

The total electron content (TEC) and electron density profiles are two key parameters in the ionosphere. Therefore, imaging the TEC and electron density profile is very crucial 
to determine the status of the ionospheric activities. In addition, the TEC in the Earth's ionosphere and plasmasphere is important for estimation and correction of propagation delays in satellite positioning, and predicting space weather and ionospheric disturbances due to geomagnetic storms and solar flares, etc [6].

In order to sufficiently and conveniently use data centers from GPS, Center for Orbit Determination (CODE), Jet Prolusion Laboratory (JPL), Polytechnical University of Catalonia (UPC) and European Space Agency (ESA) in the ionosphere to evaluate the annual mean correlations of vertical electron content by calculating and examining vertical electron content correlations for each data sets [7]. Regularized estimation of TEC (Reg-Est) is a novel technique which can combine signals from all the satellites for a given instant and given station and estimate the vertical TEC (VTEC) values for any desired period without missing any important feature in temporal or spatial domain $[8,9]$.

A number of investigations have been used one of GPS data sets in the past. While the basis of selection of one data set over the other for a given study has never been formally presented, there is a need to know their relative accuracy and advantages over each other for the sake of comparing results of past and future studies. This study is conducted to fill this information gap and assess the level of agreements between different data sets so that future studies are based on objectively selected data sets by taking in to considerations the outcome of this annual mean comparison and their correlation study.

\section{The Background Theory}

Global ionosphere maps (GIM) are generated on a daily basis at Center for Orbit Determination in Europe (CODE) that belongs to the Astronomical Institute of the University of Bern, Switzerland using data from about 200 GPS (Global Navigation Satellite System) GLONASS sites of the International GPS Services (IGS) and other institutions [10]. The file contains all the information relative to the computation process and the VTEC information are presented in the form of a grid of 2.5 degrees in latitude and 5 degrees in longitude [11].

The Total Electron Content (TEC) is one of the main characteristics of ionospheric propagation. The electron contents of the ionosphere are affected by several parameters, such as the altitude, location, time of day, season, solar activity, and solar disturbances. These factors, in turn, affect the signal propagations that travel using or through the ionosphere.

The vertical total electron content (VTEC) is modeled in a solar-geomagnetic reference frame using a spherical harmonics expansion up to degree and order 15. Piece-wise linear functions are used for representation in the time domain. The time spacing of their vertices is 2 hours, conforming to the epochs of the VTEC maps. Instrumental biases, so-called differential $P_{1}-P_{2}$ code biases (DCB), for all GPS satellites and ground stations are estimated as constant values for each day, simultaneously with the 13 times 256, or 3328 parameters used to represent the global VTEC distribution.

TEC has complex temporal-spatial fluctuations including the periodic variation, such as the diurnal variation, monthly variation, and annual variation, and the momentary disturbance [12]. The global mean TEC from the Jet Propulsion Laboratory (JPL) (1998-2008) was calculated, and the mean data expressed strong solar cycle, annual/semiannual, and 27-day variations. The annual variations were found to be stronger in the southern hemisphere, and the semiannual phases and amplitudes were much stronger in the conjugate hemisphere $[13,14]$.

The TEC measurements, the Ionospheric effect on radio wave propagation mentioned in the previous subsection is often used. TEC has been measured for decades using the Faraday rotation effect on a linear polarized propagating plane wave. But today TEC measurements are made mostly using GPS data because of the good global coverage of the GPS observation network. In general, vertical TEC can be calculated by taking the line integral of the three-dimensional electron density $\left(n_{e}\right)$ profile as a function of height (h) over the signal path from the GPS ground station to the satellite [15].

$$
T E C_{v}=\int n_{e}(h) d h
$$

Where $n_{e}$ is the total number of the accumulated electrons in each square meter area through the differential distance (dh) along the path from the satellite to the receiver on the ground. This equation is used to calculate ionosphere vertical electron content. To compute the ionosphere effects, the free electrons density along the satellite receiver propagation path should be taken into account total electron content unit (1 TECU $=10^{16} \mathrm{~m}^{-2}$ ).

The electron density delay depends on the frequency and so the utilization of two GPS signals L band of frequencies ( $f_{1}=1575.42 \mathrm{MHz}$ and $f_{2}=1227.6 \mathrm{MHz}$ ) undergo different perturbations will enable the elimination of the ionosphere delay $[16,17]$. Based on a dual frequency GPS receiver can measure the difference in ionospheric delays between the $L_{I}$ and $L_{2}$ of the GPS frequencies, which are generally assumed to travel along the same path through the ionosphere. Thus, the absolute TEC can be obtained as:

$$
P_{1}-P_{2}=40.3 \operatorname{TEC}\left(\frac{1}{{f_{2}}^{2}}-\frac{1}{f_{1}^{2}}\right)
$$

Where the time delay of the two signals are converted to pseudoranges and recorded as $P_{1}$ and $P_{2}$ signals. The TEC values can be calculated from the difference between $P_{1}$ and $P_{2}$ signals which is called the absolute TEC; the difference between $L_{1}$ and $L_{2}$ can be used to compute TEC which is referred to as relative TEC; and it is possible to compute TEC by using both $\left(L_{1}-L_{2}\right)$ and $\left(P_{2}-P_{1}\right)$ measurements and in some studies the instrumental biases are solved together with TEC estimation using a combination of $\left(P_{2}-P_{1}\right)$ and $\left(L_{1}-L_{2}\right)$ measurement [9].

$$
T E C=\frac{1}{40.3}\left(\frac{f_{1} f_{2}}{f_{1}-f_{2}}\right)\left(P_{2}-P_{1}\right)
$$


In practice, slant TEC values, which are measured along the ray path from a GPS satellite to a ground receiver, are converted to equivalent VTEC assuming the ionosphere to be compressed into a thin shell at a shell height of $\mathrm{h}$ as shown in Figure 1.

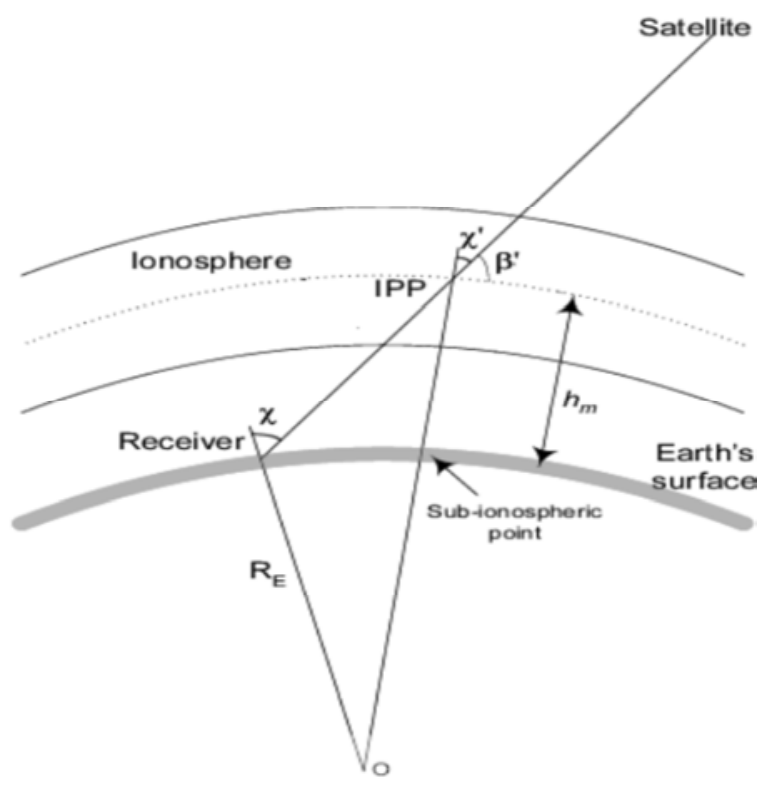

Figure 1. Geometry for conversion of slant and vertical TEC.

The vertical TEC values (VTEC) are assigned to an ionospheric pierce point, which is the intercept of the line-ofsight ray and the thin shell at the altitude $h$. Often vertical TEC (VTEC) is obtained from the slant TEC (STEC) by use of a mapping function [18] as follows:

$$
\begin{aligned}
& \text { VTEC }=\chi \times S T E C \\
& \chi=\left[1-\left(\frac{\cos ^{2} \chi}{h+R_{e}}\right)\right]^{1 / 2}
\end{aligned}
$$

Where $\chi$ is an elevation angle of a satellite, $\mathrm{h}$ is ionospheric shell height, and $\mathrm{R}_{\mathrm{e}}$ is the Earth's mean radius. This mapping function takes the curvature of the Earth into account.

\section{Data and Methodology}

A single ionospheric layer need an assumption is taken to account to convert the slant path TEC to vertical TEC with a mapping function. This work is an extension of [19] to study annual mean variation and correlation of vertical electron contents on global data centers. The international GNSS service (IGS) supplied TEC data with the time resolution of 2 hours determined from more than 350 IGS stations on a global scale. These TEC data can be used to study the annual mean correlations of VTEC of the ionosphere because of global data with the appropriate time resolution.

\section{Results and Discussion}

The annual mean VTEC included in Figures 2-6 are IGS,
COD, ESA, JPL and UPC as indicated in the figure below. In general, the amount of VTEC in the low-latitude region is maximum, and the amount of TEC in the high latitudes is minimum. The conventional total electron content is TECU where 1 TECU $=10^{16} \mathrm{el} / \mathrm{m}^{2}$. The amount of TEC near the equator is up to more than $20 \mathrm{TECU}$, which is three times greater than that at the high latitudes. On the whole, TEC should decrease progressively from the low latitudes to the high latitudes with some abnormal phenomena on local areas.

The annual mean VTEC from JPL global GPS TEC shown in Figure 5 is the highest followed by IGS global GPS TEC whereas lowest TEC is observed in COD global GPS TEC. A distinct feature exhibited by all data sets is presence of peak VTEC along equatorial latitude belt which fades out with increase in latitude. This clearly demonstrates VTEC dependence on solar zenith angle. Moreover, there is some sort of west-east asymmetry in the distribution annual mean VTEC. The center of the peak of VTEC in the western hemisphere has shown slight shift southward. In contrast, the same peak has shifted northward leading to wavy structure when seen from given latitude across the whole longitude. Perhaps, this wavy structure is linked to planetary waves of wavenumber 1 or 2 .

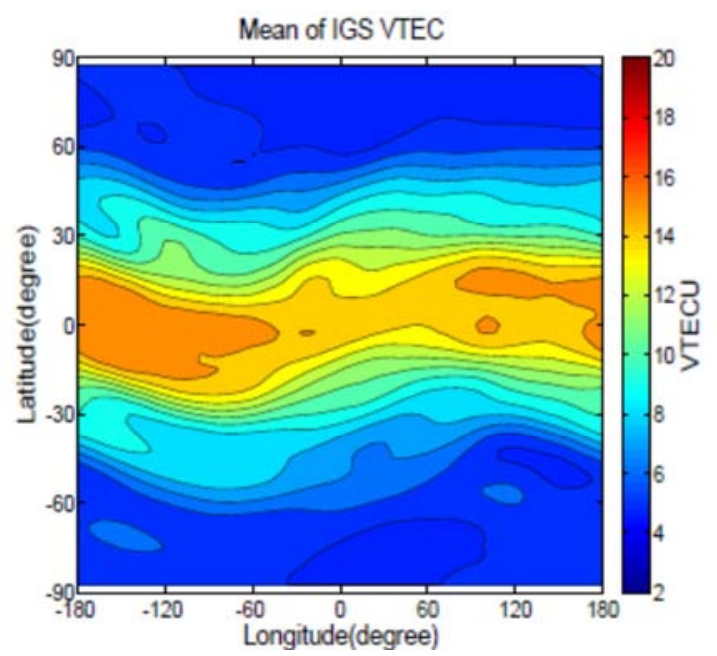

Figure 2. Annual mean IGS VTEC obtained from GPS observation in 2008.

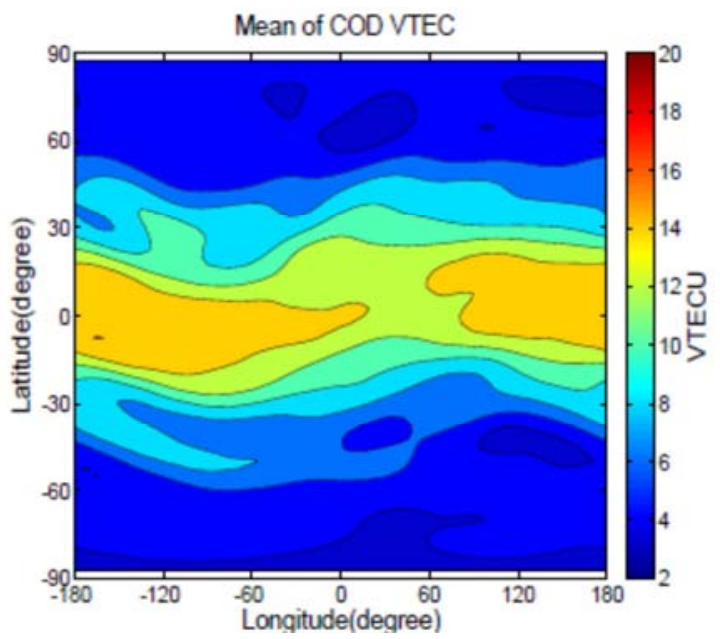

Figure 3. Annual mean COD VTEC obtained from GPS observation in 2008. 


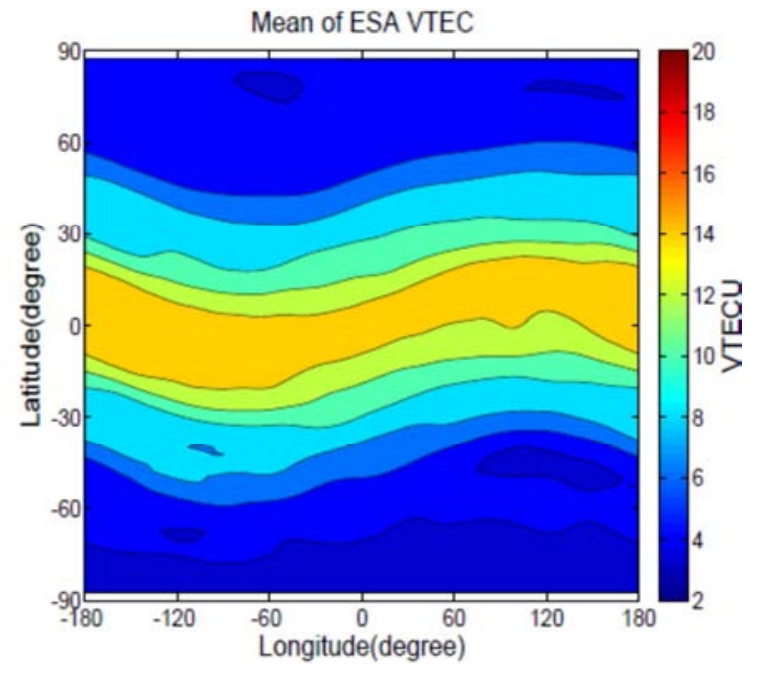

Figure 4. Annual mean ESA VTEC obtained from GPS observation in 2008.

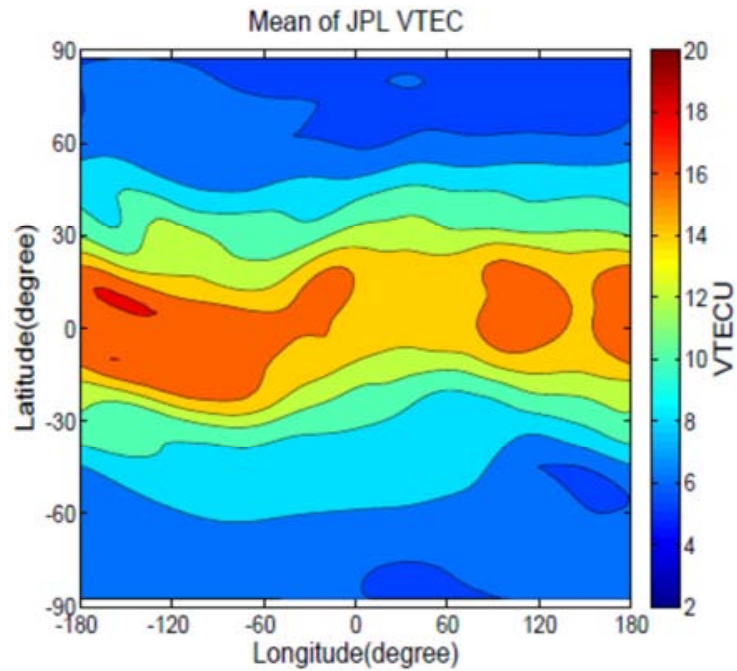

Figure 5. Annual mean JPJ VTEC obtained from GPS observation in 2008.

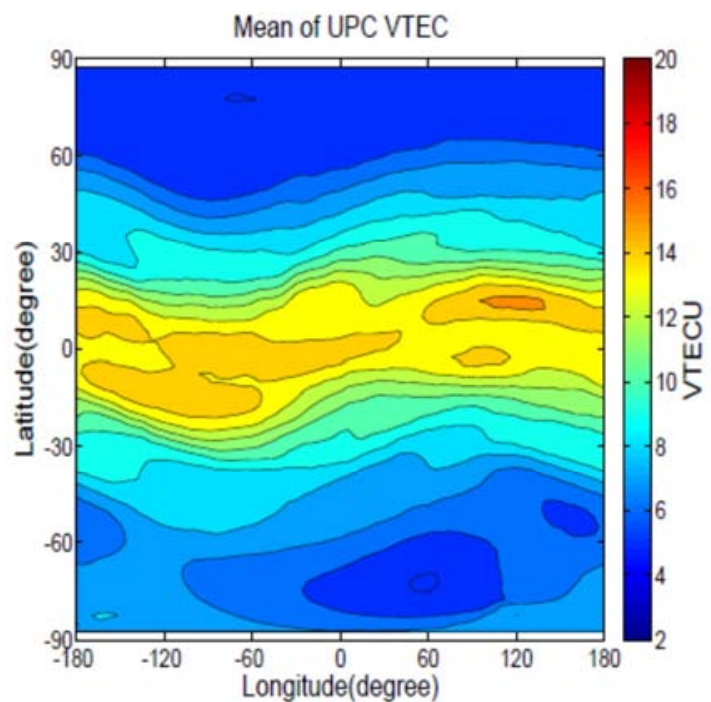

Figure 6. Annual mean UPC VTEC obtained from GPS observation in 2008.

In all cases in polar regions, the annual mean VTEC generation mechanism is different from that in the mid low latitude zones, and it is mainly caused by the ionisation effect of solar radiation, magnetosphere electric fields, electron precipitation over the polar region, aurora particle precipitation, polar plasma convection and density change of neutral atmospheric composition [20].

\subsection{Correlations}

Correlation quantifies the extent to which two quantitative variables $\mathrm{X}$ and $\mathrm{Y}$ go together. When high values of $\mathrm{X}$ are associated with high values of $Y$, a positive correlation exists. When high values of $X$ are associated with low values of $Y$, a negative correlation exists. While a perfect correlation is easy to decipher, it is difficult to guess the coefficient of weaker correlations. That is why Karl Pearson developed a precise mathematical measure of correlation known as Pearson's.

$$
r=\frac{\sum(X-\bar{X})(Y-\bar{Y})}{\sqrt{\sum(X-\bar{X})^{2}(Y-\bar{Y})^{2}}}
$$

A correlation coefficient measures the strength and direction of a linear association between two variables. The closer value is to 1 , the stronger the relationship. A correlation of zero indicates that there is no linear relationship between the variables. The coefficient can be either negative or positive.

\subsection{Correlations Maps}

Figures 7-16 show correlation of VTEC from different data centers. IGS VTEC is strong correlated with COD, JPL and UPC global VTEC as reflected by high correlation values exceeding 0.98 over tropical latitude bands. In contrast, correlation of IGS VTEC and ESA VTEC is very poor with values less than 0.90 . Similar relationship between other VTEC is noted. For example, correlations of COD VTEC with JPL and UPC global VTECs are strong. However, COD has weak correlation with ESA VTEC suggesting that ESA VTEC is different from most of other GPS VTEC in this study. It is straightforward from Figure 16 that JPL and UPC VTEC are strongly correlated.

As noted above, the agreements between the different VTECs from the five data centers are reasonably good despite relative difference among them. This suggests consistency of the data processing and common data sources used in each case. The relative advantage as reflected in very high correlation between VTEC data from some centers arises likely from difference in the number of GPS used and the mapping algorithm. A good example of such discrepancies can be noted from poor correlations in Figures $7-16$ at $60^{\circ} \mathrm{S}$ and $120^{\circ} \mathrm{W}$ for IGS versus ESA, COD versus ESA, JPL versus ESA and UPC versus ESA. This is probably related to ESA VTEC data quality which depends on aforementioned factors. Similar poor correlations are observed close to polar region in the western hemisphere between COD and UPC as well as JPL and UPC. However, the cause of this is likely the result of sparse GPS network over southern hemisphere as compared to northern hemisphere which has dense network due to landmass. 


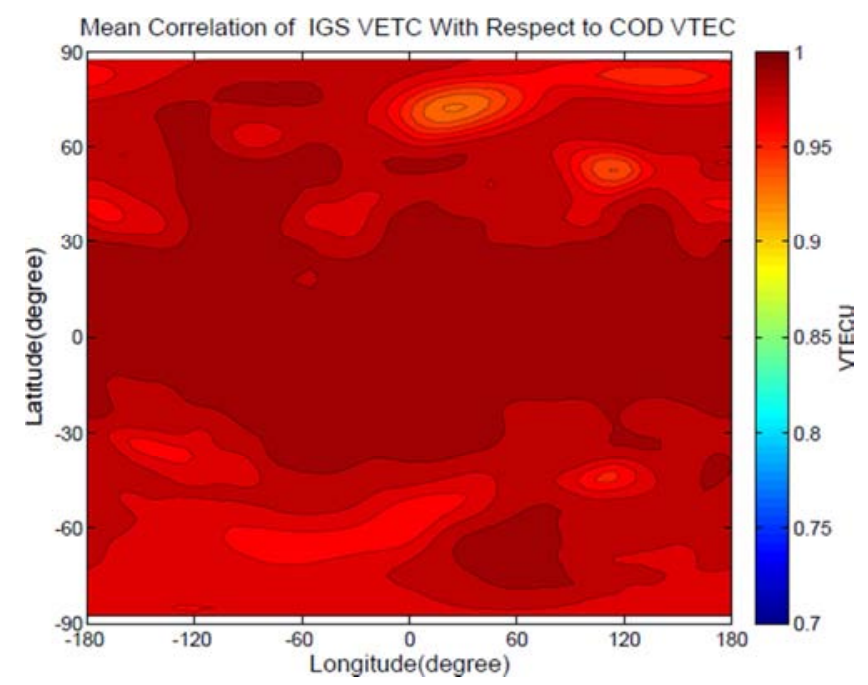

Figure 7. Mean correlation of IGS VTEC with respect to COD VTEC obtained from GPS observation in 2008

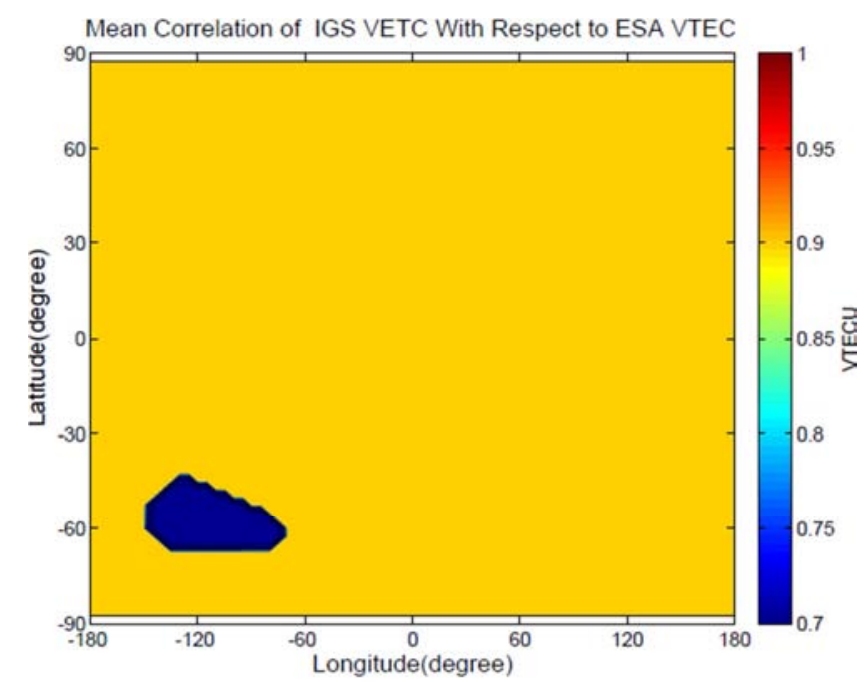

Figure 8. Mean correlation of IGS VTEC with respect to ESA VTEC obtained from GPS observation in 2008.

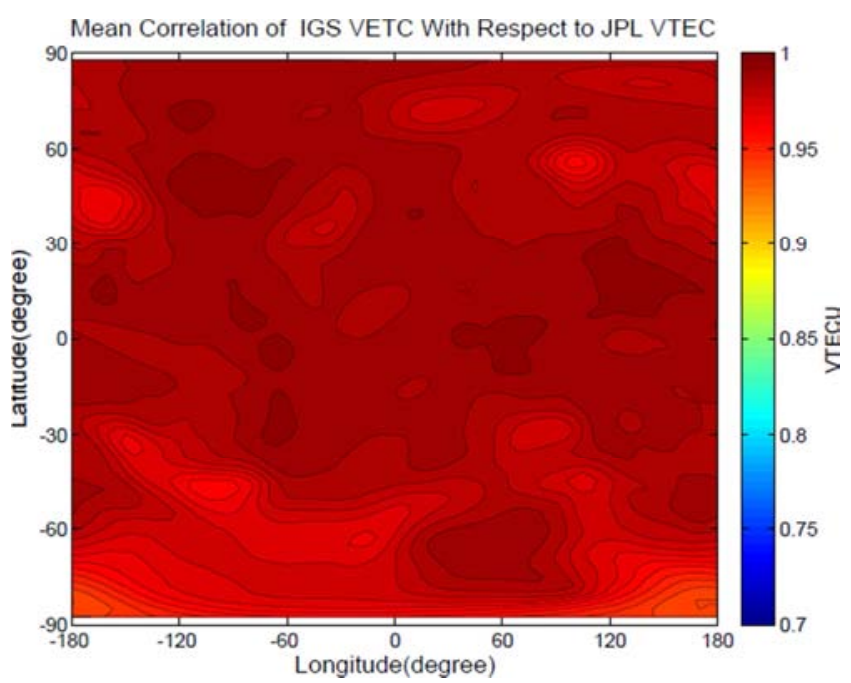

Figure 9. Mean correlation of IGS VTEC with respect to JPL VTEC obtained from GPS observation in 2008.

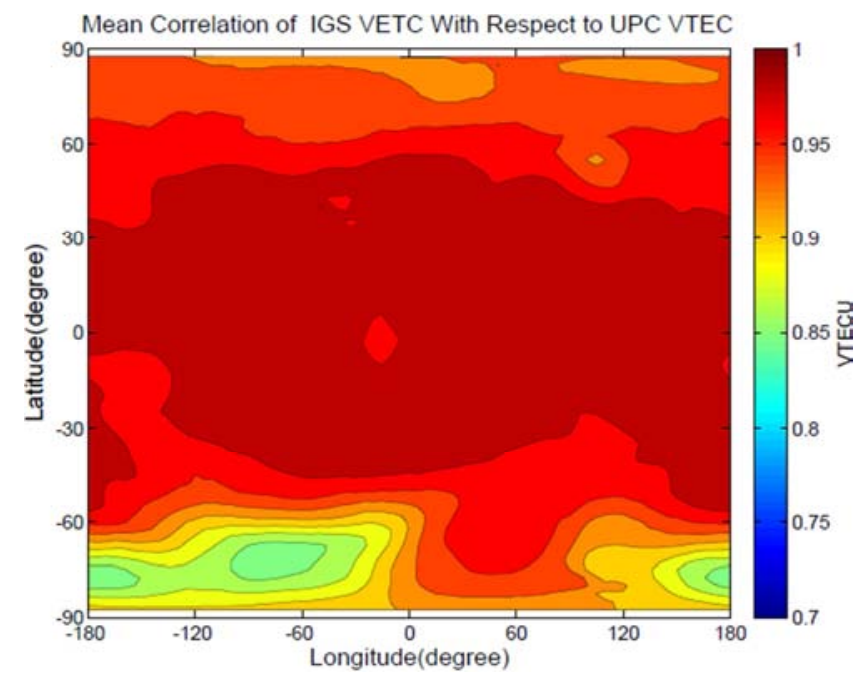

Figure 10. Mean correlation of IGS VTEC with respect to UPC VTEC obtained from GPS observation in 2008.

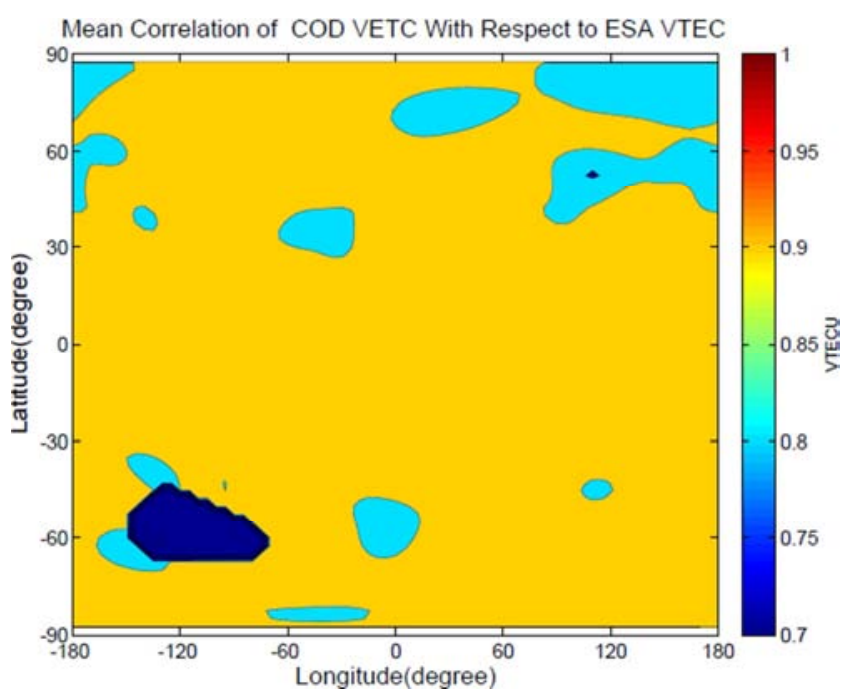

Figure 11. Mean correlation of COD VTEC with respect to ESA VTEC obtained from GPS observation in 2008.

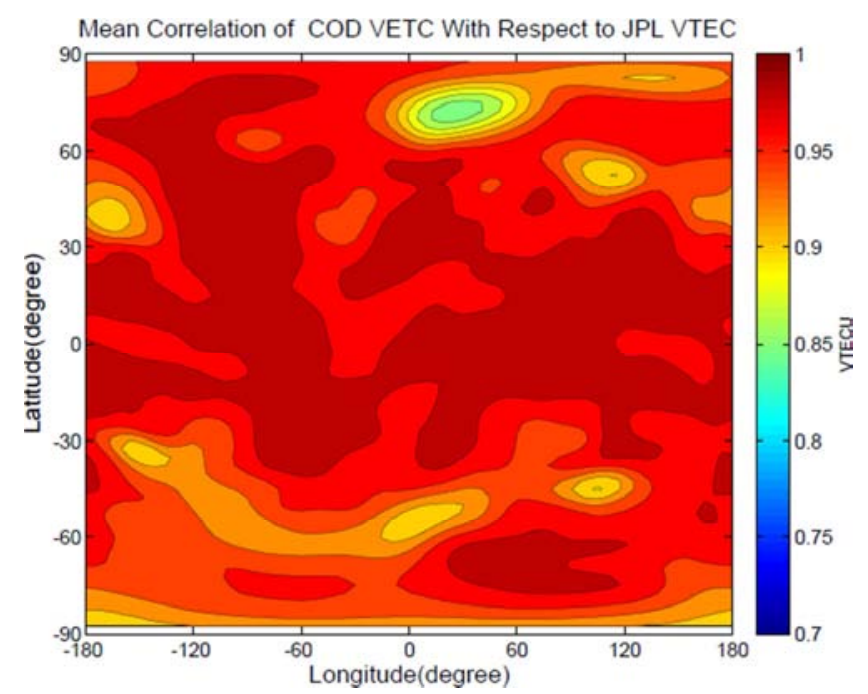

Figure 12. Mean correlation of COD VTEC with respect to JPL VTEC obtained from GPS observation in 2008. 


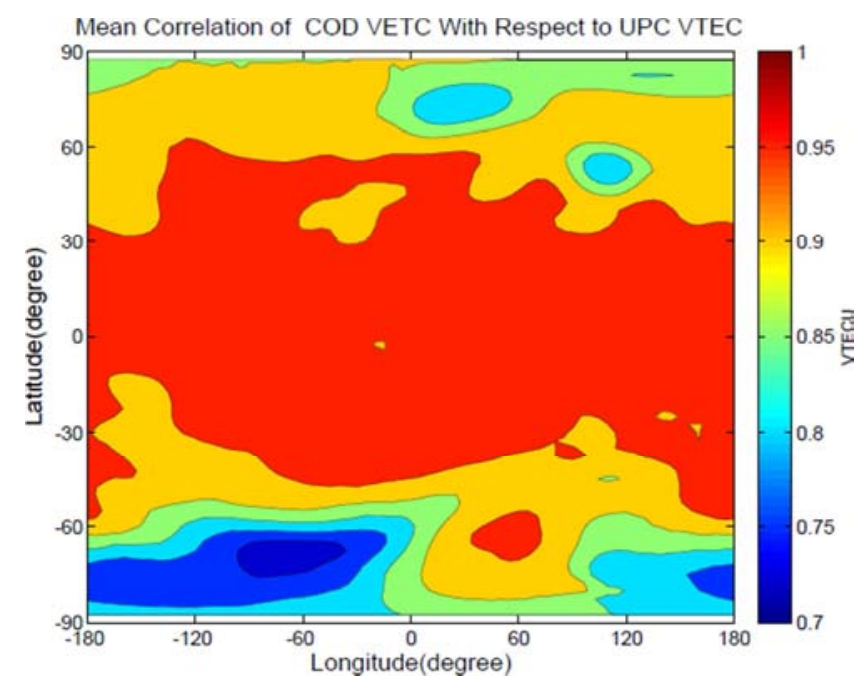

Figure 13. Mean correlation of COD VTEC with respect to UPC VTEC obtained from GPS observation in 2008.

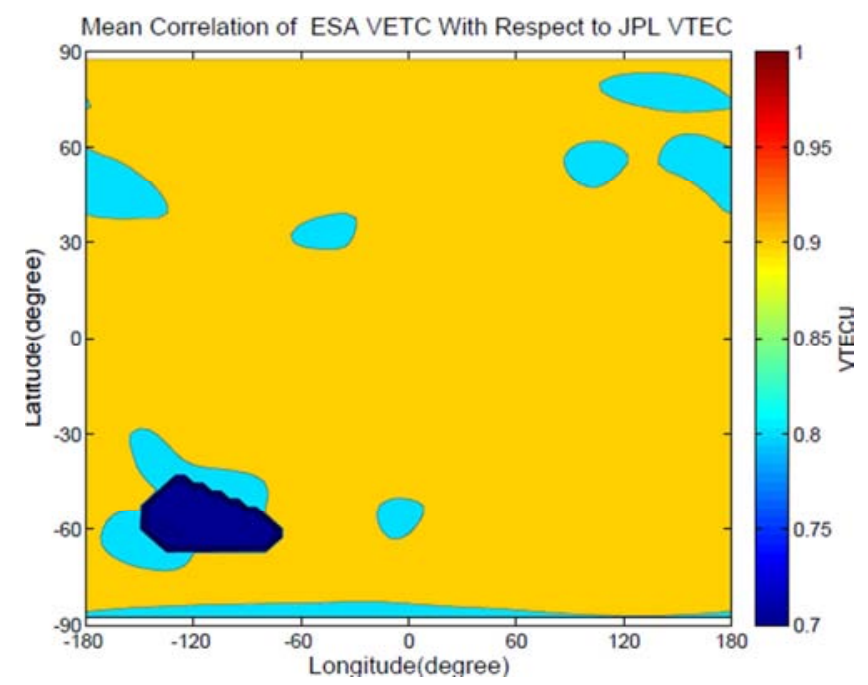

Figure 14. Mean correlation of ESA VTEC with respect to JPL VTEC obtained from GPS observation in 2008.

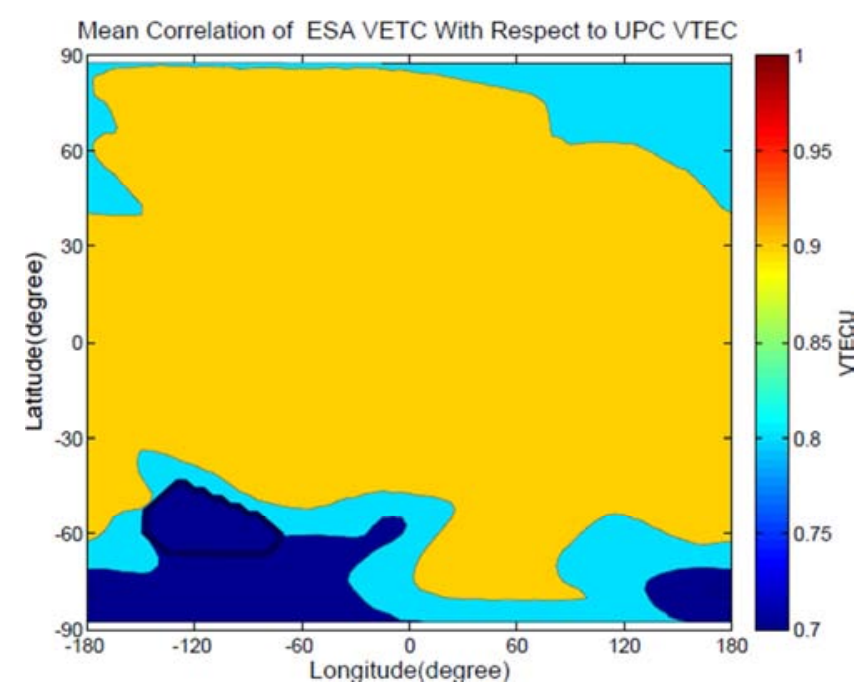

Figure 15. Mean correlation of ESA VTEC with respect to UPC VTEC obtained from GPS observation in 2008.

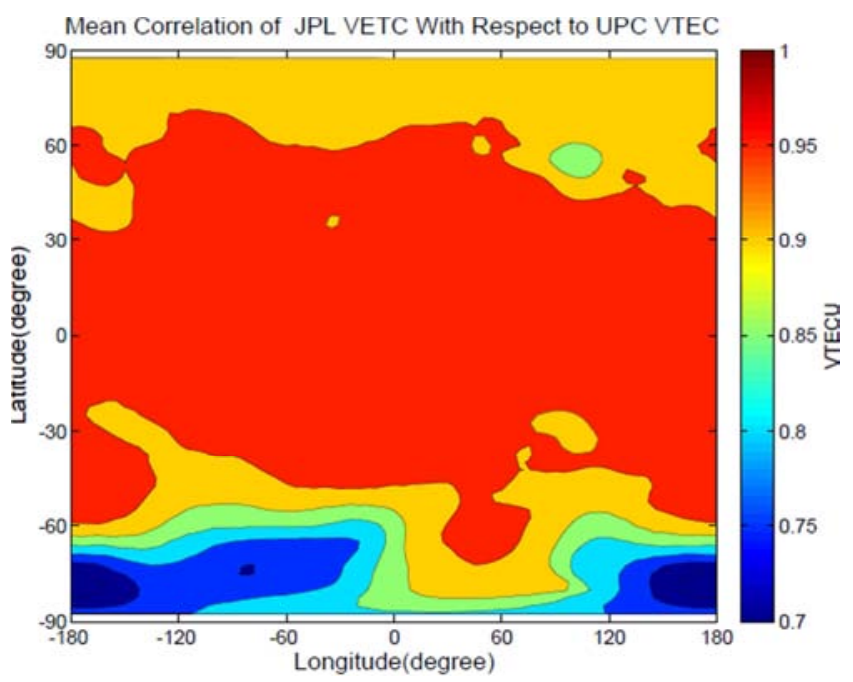

Figure 16. Mean correlation of JPL VTEC with respect to UPC VTEC obtained from GPS observation in 2008.

\section{Conclusions}

In this study we have used TEC observations for 2008 from globally distributed GPS receivers to describe the annual mean VTEC variation in the ionosphere at different time scales. The gridded global TECs are available from IGS, COD, ESA, JPL and UPC data centers. The inter comparison between the data sets show that generally ESA VTEC is higher than all of them, followed by JPL, IGS, COD and UPC VTEC respectively.

The agreements between the different VTECs from the five data centers are reasonably good despite relative difference among them. This suggests consistency of the data processing and common data sources used in each case. The relative advantage as reflected in very high correlation between VTEC data from some centers arises likely from difference in the number of GPS used and the mapping algorithms. Poor correlations observed close to polar region in the western hemisphere between COD and UPC as well as JPL and UPC might be linked to sparse GPS network over this region.

\section{References}

[1] Ivanov-Kholodny, G. S., Nikolsky, G. M., 1969. The sun and ionosphere. Nauka, Moskow.

[2] Akasofu S.-I, Chapman S. (1972), Solar Terrestrial Physics. Oxford University Press, Oxford alan N, Bailey GJ, Abdu MA, Oyama KI, Richards PG, MacDougall J, Batista IS (1997) Equatorial plasma fountain and its effects over three locations: evidence for an additional layer, the F3 layer. J Geophys Res 101: 20472056.

[3] Krinberg, I. A., Taschilin, A. V., 1984. Ionosphere and Plasmosphere. Nauka, Moscow. Leitinger, R., Zhang, M., Radicella, S. M., 2005. An improved bottomside for the ionospheric electron density model NeQuick, Annals of geophysics 48 (3), 525-534.

[4] Bryunelli, B. E., Namgaladze, A. A., (1988). Physics of the Ionosphere. Nauka, Moscow. 
[5] DasGoupta, A, Paul A., and Das A., (2007), Ionospheric total electron content studies with GPS in the equatorial region, Indian Journal of Radio and Space physics, 36, 278-293.

[6] Kelley, Michael C.(1989), The Earths Ionosphere: Plasma Physics and Electrodynamics, Academic Press.

[7] SCHAER, S.; Markus; R. Gerhard; B. Timon, A. S., (1996). Daily Global Ionosphere Maps based on GPS Carrier Phase Data Routinely produced by the CODE Analysis Center, Proceeding of the IGS Analysis Center Workshop, Silver Spring, Maryland, pp. 181-192, USA.

[8] Arikan, F., Erol, C. B., Arikan, O (2003). Regularized estimation of vertical total electron content from Global Positioning System data. J. Geophys. Res.-Space Phys. 108 (A12), 1469-1480.

[9] Arikan, F., Arikan, O., Erol, C. B., (2007), Regularized estimation of TEC from GPS data for certain mid latitude stations and comparison with the IRI model, Advances in Space Research, 39, 867-874.

[10] Guo, J, Li W, Liu X, Kong Q, Zhao C, Guo B., (2015). Temporal-Spatial Variation of Global GPS Derived Total Electron Content, 1999-2013. PLoS ONE 10 (7): e0133378. Doi: 10.1371 /journal.

[11] Natali, M. P. and Meza A, (2011). Annual and semiannual variations of vertical total electron content during high solar activity based on GPS observations, Ann. Geophysics., 29, 865-873.

[12] Xu, JS, Li XJ, Liu YW, Jing M., 2003. Effects of declination and thermospheric wind on TEC longitude variations in the mid-latitude ionosphere. Chinese Journal of Geophysics; 56: 1425-1434. doi: 10.6038/cjg20130501.

[13] Mallis, M, Essex EA., (1993). Diurnal and seasonal variability of the southern-hemisphere main ionospheric trough from differential-phase measurements. Journal of Atmospheric and Terrestrial Physics; 55: 1021-1037.

[14] Liu LB, Wan WX, Ning BQ, Zhang ML, (2009). Climatology of the mean total electron content derived from GPS global ionospheric maps. Journal of Geophysical Research; 114: A06308. doi: 10.1029/2009JA014244.

[15] Bothmer, V. and I. A. Daglis (2007), Space Weather: Physics and Effects, 213, 315-318, 438 pp., Praxis Publishing Ltd., Chichester, UK.

[16] Klobuchar, J. A., (1996). Global Positioning System: Theory and Applications. American Institute of Aeronautics and Astronautics, Inc., Washington DC.

[17] Boutiouta S. and Belbachir A. H, (2006). Magnetic Storms Effects on the Ionosphere TEC through GPS Data, Information Technology Journal 5 (5), 908-915, DOI: $10.3923 / \mathrm{itj} .2006 .908 .915$.

[18] Mannucci, A. J., B. D. Wilson, and C. D. Edwards (1993), A new method for monitoring the Earth's ionospheric total electron content using the GPS global network, in Proceedings of ION GPS-93, the 6th International Technical Meeting of the Satellite Division of The Institute of Navigation, Salt Lake City, UT, 22-24 September, pp. 1323-1332, The Institute of Navigation, Alexandria, Va.

[19] Shambel Gizachew, Belay Sitotaw, Gizaw Mengistu. Comparison of Global Vertical Total Electron Content from Various Global Data Centers. International Journal of Astrophysics and Space Science. Vol. 7, No. 4, 2019, pp. 5057. doi: 10.11648/j.ijass.20190704.12.

[20] Zhu, AQ., (2008). Comparative study of polar ionospheric F layer in both hemispheres. Master thesis, Xi Dian University. Available: http://www.docin.com/p-119889 148.html. 